\title{
Lithium and Rotation in Pop I stars
}

\author{
Sushma V. Mallik \\ Indian Institute of Astrophysics, Bangalore 560034, India
}

\begin{abstract}
Lithium abundances have been determined for 130 stars sampled on the basis that they have just about completed depletion of $\mathrm{Li}$ on the main sequence and have not yet begun their ascent on the red giant branch. The main goal is to study the effects of rotation on Li depletion. Analysis of the data reveals that fast rotating stars have suffered little depletion whereas slowly rotating stars have both low and high $\mathrm{Li}$ abundances. No one-to-one correlation appears to exist between $\mathrm{Li}$ abundance and the present $v$ sini. The large observed spread in $\mathrm{Li}$ abundance is perhaps a consequence of an initial range in rotational velocities in stars spinning down to low values on the main sequence. An attempt is made to study the $\mathrm{Li}$ evolution as a function of age, mass and rotation against the backdrop of the evolutionary tracks on the HR diagram.
\end{abstract}

\section{Introduction}

Lithium is a fragile element burning at only a few million degrees through the reaction ${ }^{7} \mathrm{Li}(p, \alpha){ }^{4} \mathrm{He}$ and therefore an excellent probe of physical processes occurring inside stars. In a typiçal main sequence star, Li survives in the outer $40 \%$ of the stellar radius and is depleted inside of it. Standard stellar models do not predict depletion of $\mathrm{Li}$ in the MS stars (except the coolest dwarfs of $T_{\text {eff }}$ $<4000 \mathrm{~K}$ ) because the convection zone only lies within the outer $10 \%$ of the stellar radius in the much shallower and cooler region than the Li preservation boundary. However, there is enough observational evidence now to suggest that $\mathrm{Li}$ depletion is a rule, rather then the exception in $\mathrm{F}$ and $\mathrm{G}$ dwarfs (Duncan 1981; Balachandran 1990; Randich et al. 1995, 1999; Lebre et al. 1999). In fact, a vast majority of them are severely depleted in lithium, in contrast to the predictions of the standard stellar model calculations which include only convective mixing. In such a model of say an $\mathrm{F}$ star, the $\mathrm{Li}$ abundance goes down a little from $\log \mathrm{N}(\mathrm{Li})$ around 3.3 to 2.7. This is understood to be a consequence of the destruction of $\mathrm{Li}$ in the pre-MS phase when the base of the convection zone is deep and hot enough to destroy Li (D'Antona \& Mazzitelli 1994).

It is clear that the stellar models which ignore possible effects due to rotation, diffusion, mass loss, magnetic fields etc. fail to reproduce the observed $\mathrm{Li}$ abundance patterns in $\mathrm{F}$ and $\mathrm{G}$ dwarfs and that some additional physics is occurring inside these stars as emphasized by Deliyannis, Pinsonneault \& Charbonnel (2000). Several non-standard processes have been investigated in the past in order to explain such large spreads in Li abundance and large depletions in many of them. The present study was undertaken to explore, in particular, the effects of rotation on the depletion of $\mathrm{Li}$ in $\mathrm{F}$ and $\mathrm{G}$ stars. A fresh look at this problem was triggerred off by two recent observational developments. The 

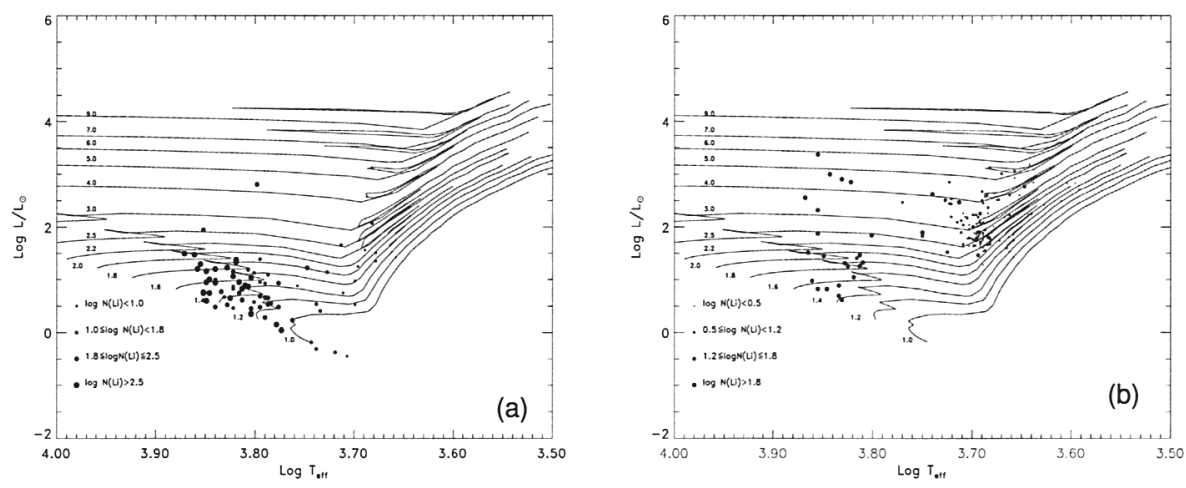

Figure 1. Stars chosen on the basis of their spectral classification on the HR diagram a) dwarfs and subgiants b) giants. Evolutionary tracks of Girardi et al. (2000) are shown for masses ranging from 1.0 to $7.0 \mathrm{M}_{\odot}$

first is the catalogue of rotational velocities measured with high precision in a large number of F, G and K stars by de Medeiros \& Mayor (1999). The other is the Hipparcos Catalogue, a single source which gives parallaxes with accuracy of $\sim 1$ milliarcsec for distances $\leq 100 \mathrm{pc}$. Thus one can actually trace the evolution of $\mathrm{Li}$ abundance of a star on the HR diagram against the backdrop of the evolutionary tracks as a function of mass, age and rotation.

\section{Observations}

Based on their spectral classification, a sample of $235 \mathrm{~F}, \mathrm{G}$ and $\mathrm{K}$ dwarfs, subgiants and giants was selected from the Bright Star Catalogue, the $[\mathrm{Fe} / \mathrm{H}]$ Catalogue (Cayrel de Strobel et al. 1997) and de Medeiros \& Mayor Catalogue spanning a large range in $[\mathrm{Fe} / \mathrm{H}]$ and $v$ sini. CCD spectra of these stars were obtained in the Li region at a spectral resolution of $\sim 0.35 \AA$ using the coude echelle spectrograph at the 102cm telescope at the Vainu Bappu Observatory in Kavalur. With the measured Li EQWs as the input and an appropriate choice of models based on the stellar parameters from the grid of model atmospheres due to Gustafsson et al. (1975) generated by Luck (1992), Li abundances were determined using MOOG (Sneden 1973, upgraded 2001). The plot of dwarfs and subgiants shown in Fig.1a on the HR diagram superposed against evolutionary tracks of Girardi et al. (2000) reveals that some of them have already begun their ascent up the red giant branch (RGB). Similarly, when giants are plotted on the HR diagram (Fig. 1b), it is seen that several of them are still on the subgiant branch and some are lying close to the main sequence. Stars lying on the RGB were discarded and a sample of 135 stars was selected based on their location on the HR diagram which is consistent from the point of $\mathrm{Li}$ evolution in the sense that they are completing MS depletion or have just about completed it and some of them are undergoing subgiant dilution but none have evolved far enough to have suffered depletion on the RGB. 


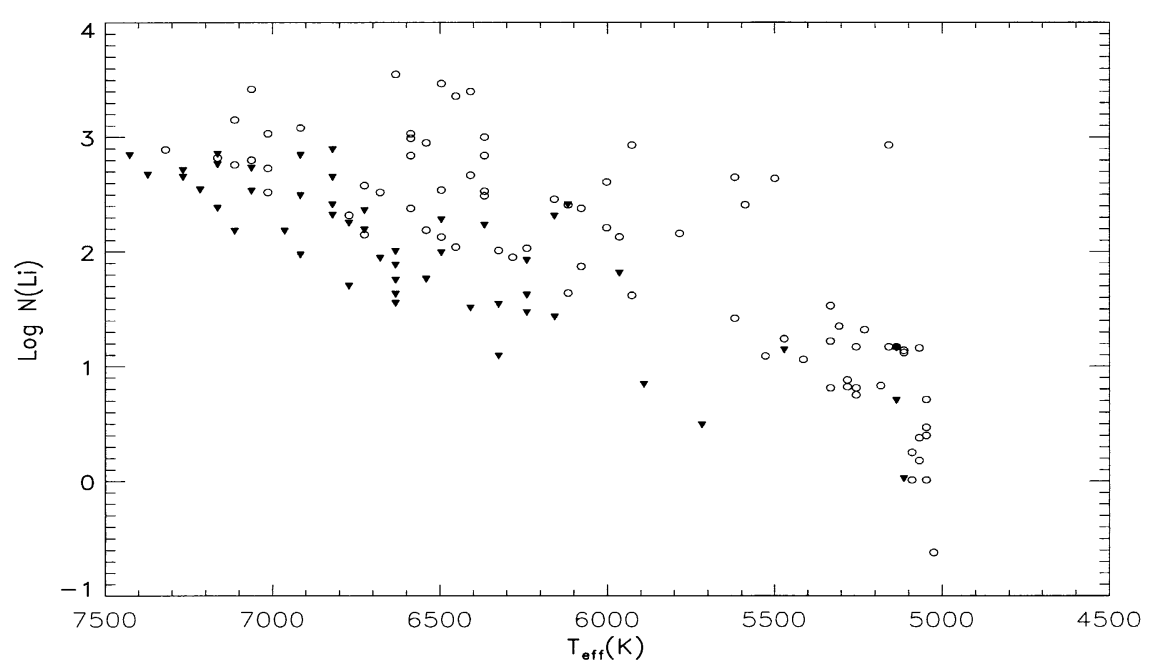

Figure 2. Li abundance vs. effective temperature. Inverted triangles denote stars with upper limits to lithium abundance

\section{Interpretation}

Li abundances of these stars plotted against effective temperature (Fig.2) suggest a fairly sudden drop at around $5300-5400 \mathrm{~K}$ which marks the onset of dilution on the subgiant branch due to the deepening of the convective envelope. One notices this process is rather fast in terms of time and the range of $T_{\text {eff }}$. The inverted triangles denote stars with upper limits to $\mathrm{Li}$ abundance. There is a large spread in $\mathrm{Li}$ for $T_{\text {eff }}=6400-6800 \mathrm{~K}$ which corresponds to the $\mathrm{Li}$ dip first discovered by Boesgaard \& Tripicco (1986). A more striking feature is the large spread in $\mathrm{Li}$ abundance seen in stars to the right of this and to the left of the drop at $5300 \mathrm{~K}$ which suggests main sequence depletion is indeed occurring in stars. The plot of their rotational velocities against $T_{\text {eff }}$ (Fig.3) shows a rotational discontinuity at $\sim 6400 \mathrm{~K}$ to the right of which stars have spun down to low values, lower than $20 \mathrm{~km} \mathrm{~s}^{-1}$ and stars to the left display a large range in $v$ sini. So the stars between 6400 and $5400 \mathrm{~K}$ (late F and early G stars) that display a large spread in Li have already spun down. On the other hand, early F stars perhaps because of their thinner convective envelopes retain more or less their intrinsic range in $v$ sini.

In Fig. 4 these stars are plotted on the HR diagram superposed on evolutionary tracks of masses ranging from 1.0 to $7.0 \mathrm{M}_{\odot}$. The increasing size of the circles denotes higher $v$ sini. Lithium evolution is better understood with more bins in $\mathrm{Li}$ abundance. Here, however, only $2 \mathrm{Li}$ abundance bins are shown; the open circles denote stars with $\log \mathrm{N}(\mathrm{Li}) \geq 2.0$ and filled circles are stars with $\log \mathrm{N}(\mathrm{Li})<2.0$. It is obvious from this figure that stars of lower masses ( $\leq$ $1.3 \mathrm{M}_{\odot}$ ) have already spun down to lower values. On the other hand, the more massive stars do so only towards the end of the subgiant branch. What is worth 


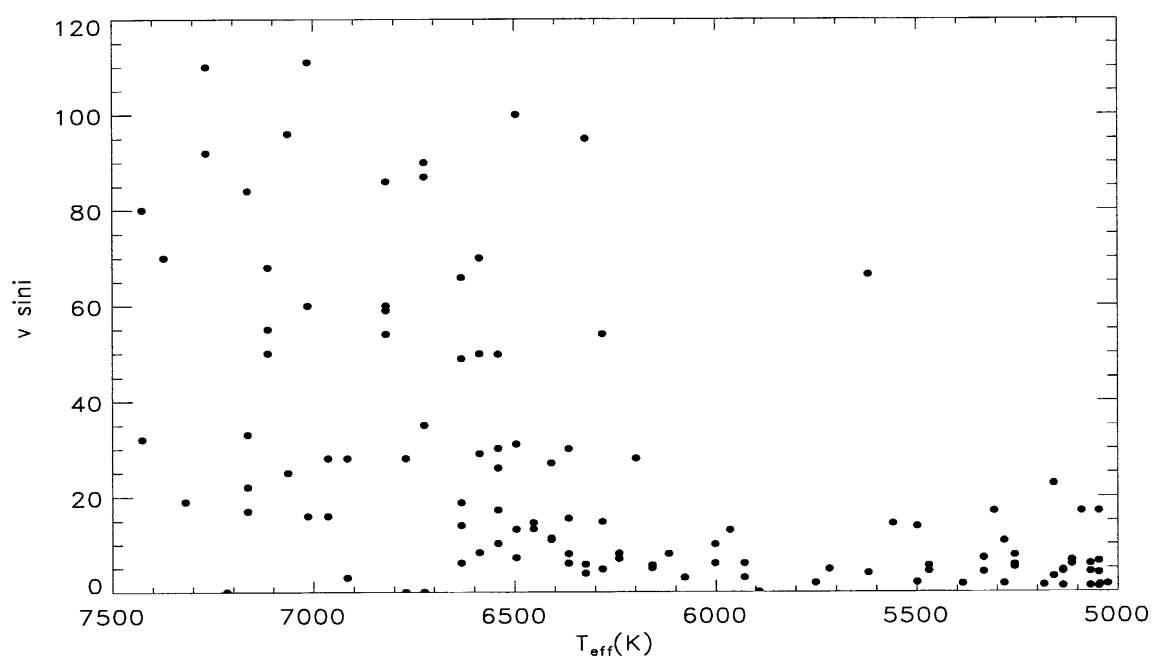

Figure 3. $\quad v$ sini vs. effective temperature

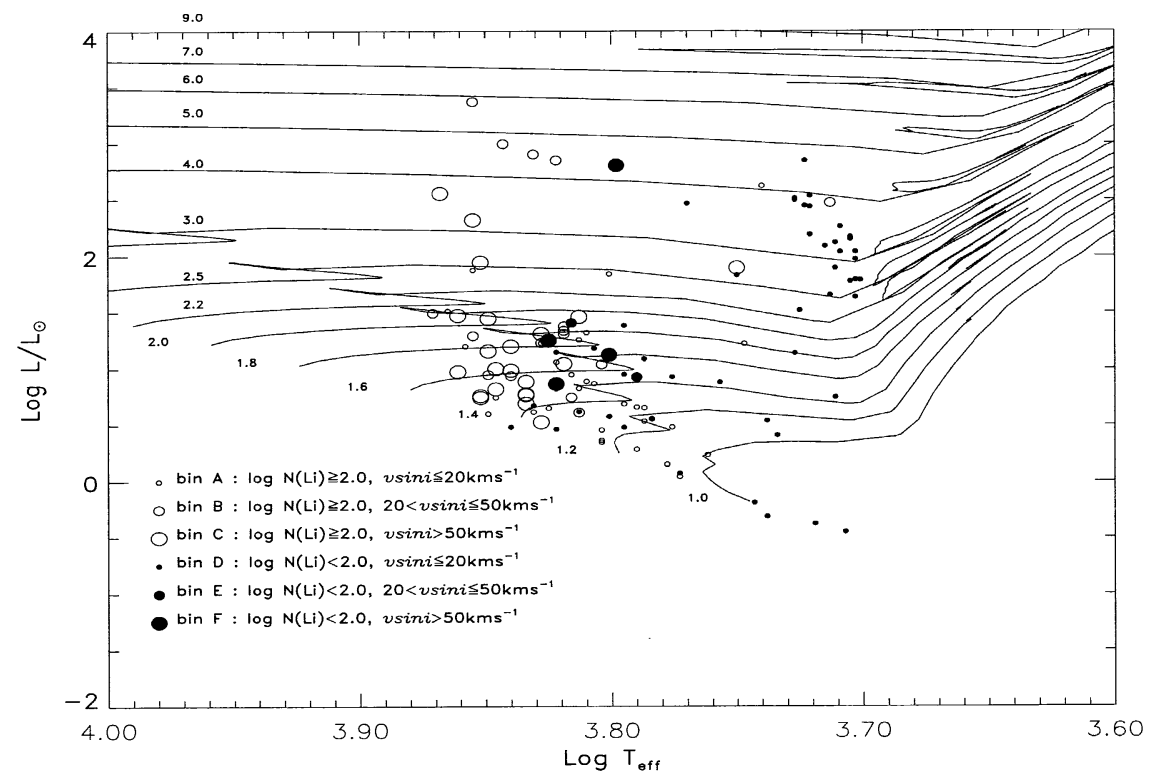

Figure 4. HR diagram of the observed stars. Symbols of increasing size denote increasing $v$ sini. Unfilled circles denote $\log \mathrm{N}(\mathrm{Li}) \geq 2.0$ while the filled ones are with $\log \mathrm{N}(\mathrm{Li})<2.0$. Also shown are theoretical evolutionary tracks of Girardi et al. (2000) for stars of initial masses ranging from 1.0 to $7.0 \mathrm{M}_{\odot}$ 


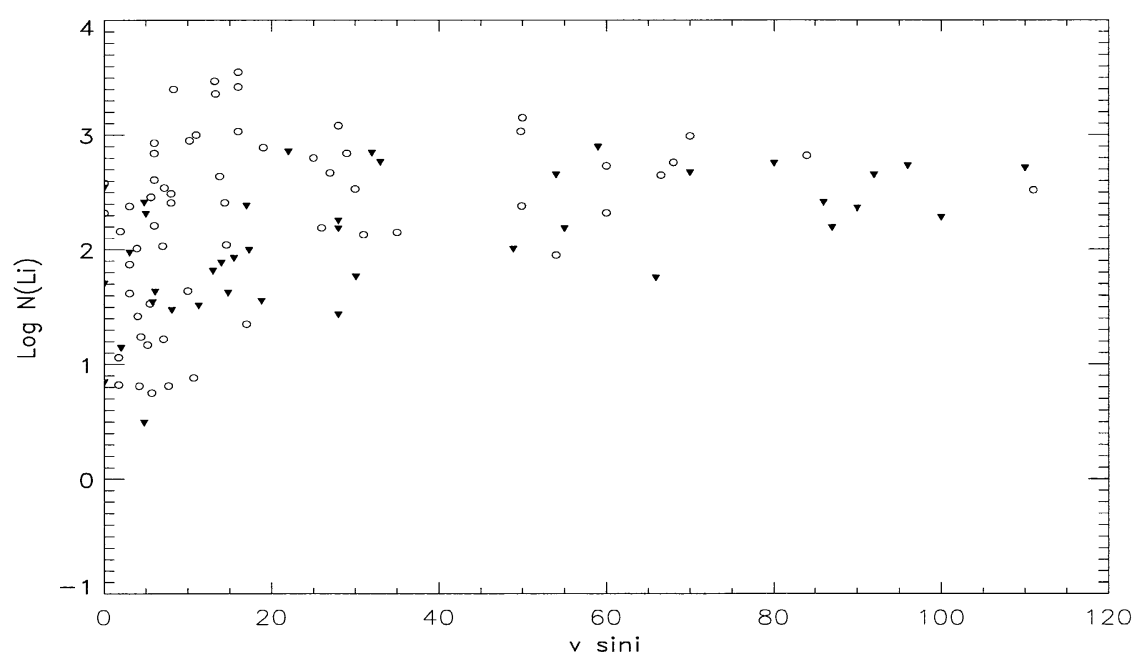

Figure 5. Li abundance vs. $v$ sini for stars of $T_{\text {eff }} \geq 5300 \mathrm{~K}$. Inverted triangles are stars with upper limits to lithium abundance

noting here is that there is a large spread in $\log \mathrm{N}(\mathrm{Li})$ for stars of low $v$ sini for all masses whereas for stars of high $v$ sini and larger masses, $\log \mathrm{N}(\mathrm{Li})$ tends to have only higher values. This is true even if the stars in the Li dip are not considered. This is more clearly demonstrated in Fig.5 where $\log \mathrm{N}(\mathrm{Li})$ is plotted against $v$ sini. Here stars of $T_{\text {eff }} \leq 5300 \mathrm{~K}$ which have already started undergoing subgiant dilution have been excluded. This trend seems to be dictated by the idea that stars which are still fast rotators have suffered little loss of angular momentum and hence have hardly undergone any mixing and hence have retained their initial Li. Among the slowly rotating stars, those born as slow rotators tell the same story but those born as fast rotators have suffered much larger losses of angular momentum and therefore have undergone more effective mixing and hence larger Li depletion. There does not seem to be a one-to-one correlation between $\mathrm{Li}$ abundance and the present projected rotational velocity. The above correlation may perhaps be driven by the initial rotational velocity the star is endowed with. It is tempting to think of a scenario where a group of stars with, say, the same initial $\log \mathrm{N}(\mathrm{Li})$ and a range in $v$ sini as they arrive on the main sequence exhibit a large scatter in $\mathrm{Li}$ abundance after they have all spun down.

Such an interpretation of the present data by no means proves that mixing induced by differential rotation is the only viable mechanism that explains large scatter in Li abundances in stars otherwise similar. An attempt has been made to explore whether such a mechanism is supported by observations and it does not appear to be at odds with them. However, the pattern as we see it in Fig.6 has to be interpreted with caution. The inverted triangles are stars with upper limits to Li abundance. The lower envelope running from high $\mathrm{Li}$ and high $v$ sini to low Li and low $v$ sini appears to skirt these upper limits. This perhaps arises because the minimum detectable EQW of the Li line increases for an obvious 
reason with increasing $v$ sini. On the other hand, the upper boundary is almost independent of $v$ sini. It would be worth increasing the sample still more to make sure whether the lower envelope has a real physical basis or is an artifact of the limited nature of the sample. Either way, it lends a strong statement on the link between $\mathrm{Li}$ abundance and rotation.

\section{References}

Balachandran, S. 1990, ApJ 354, 310

Boesgaard, A.M., Tripicco, M.J. 1986, ApJ 302, L49

Cayrel de Strobel, G., Soubiran, C., Friel, E.D., Ralite, N., Francois, P. 1997, A\&ApS 124,299

De Medeiros, J.R., Mayor, M. 1999, A\&ApS 139, 443

D’Antona, F., Mazzitelli, I. 1994, ApJS 90, 467

Deliyannis, C.P., Pinsonneault, M.H., Charbonnel, C. 2000, IAU Symp.198 on The Light Elements and their Evolution, eds. L. da Silva, M. Spite, J.R. de Medeiros (published by ASP), p.61

Duncan, D.K. 1981, ApJ 248, 651

ESA 1997, The Hipparcos \& Tycho Catalogues, ESA SP-1200

Girardi, L., Bressan, A., Bertelli, G., Chiosi, C. 2000, A\&ApS 141, 371

Gustafsson, B., Bell, R.A., Eriksson, K., Nordlund, A. 1975, A\&Ap 42, 407

Lebre, A., de Laverny, P., de Medeiros, J.R., Charbonnel, C., da Silva, L. 1999, A\&Ap 345,936

Luck, R.E. 1992, private communication

Randich, S., Pallavicini, R., Pasquini, L., Gratton, R. 1995, Mem. Soc. Astron. Italia 66,387

Randich, S., Gratton, R., Pallavicini, R., Pasquini, L., Caretta, E. 1999, A\&Ap 348, 487

Sneden, C.A. 1973, Ph.D. Thesis, The University of Texas, Austin 